\title{
Extended theorems for signal induction in particle detectors VCI 2004
}

\author{
W. Riegler* \\ CERN, PH Division, Rt. De Meyrin, Geneva 23CH-1211, Switzerland
}

Available online 13 August 2004

\begin{abstract}
Most particle detectors are based on the principle that charged particles leave a trail of ionization in the detector and that the movement of these charges in an electric field induces signals on the detector electrodes. Assuming detector elements that are insulating and electrodes with infinite conductivity one can calculate the signals with an electrostatic approximation using the so-called 'Ramo theorem'. This is the standard way for the calculation of signals e.g. in wire chambers and silicon detectors. In case the detectors contain resistive elements, which is, e.g. the case in resistive plate chambers or underdepleted silicon detectors, the time dependence of the signals is not only given by the movement of the charges but also by the time-dependent reaction of the detector materials. Using the quasistatic approximation of Maxwell's equations we present an extended formalism that allows the calculation of induced signals for detectors with general materials by time dependent weighting fields. As examples, we will discuss the signals in resistive plate chambers and underdepleted silicon detectors.
\end{abstract}

(C) 2004 Elsevier B.V. All rights reserved.

PACS: Signal theory; Particle detectors; RPCs; Silicon detectors

Keywords: 29.40

\section{Introduction}

The currents induced on grounded electrodes by moving charges can be calculated with static weighting fields using Ramo's theorem [1]. In case the electrodes are not grounded but connected with reactive elements, the voltage and current can

\footnotetext{
*Tel.: + 41-22-767-7585.

E-mail address: werner.riegler@cern.ch (W. Riegler).
}

be calculated by time-dependent weighting fields as shown in Ref. [2]. In case the volume between the electrodes contains conductive material in addition, which is the case for e.g. resistive plate chambers or underdepleted silicon detectors, we need a further extension of the theorem, which is the subject of this paper. This problem was already discussed in Ref. [3], for this conference a more practical 'recipe' formulation of the theorems is presented. 


\section{Formulation of the problem}

We investigate the scenario shown in Fig. 1. Electrodes of infinite conductivity are embedded in a medium with general position and frequencydependent permittivity $\varepsilon(\vec{x}, s)$ and conductivity $\sigma(\vec{x}, s)$ and are in addition connected with arbitrary discrete elements $(s=\mathrm{i} \omega$ is the Laplace parameter). At $t=0$ a pair of charges $q,-q$ is created at position $\vec{x}_{1}(t=0)=\vec{x}_{2}(t=0)$ from where they move along trajectories $\vec{x}_{1}(t)$ and $\vec{x}_{2}(t)$. What are the voltages $V_{n}(t)$ induced on the electrodes by the movement of these charges?

\section{Quasistatic approximation, weighting potentials, admittance matrix}

In case we can neglect Faraday's law, i.e., in case the electric fields induced by the time dependence of magnetic fields can be neglected with respect to the electric fields due to the presence of charges, Maxwell's equations (written in the Laplace domain) collapse into the equation [4,5]

$$
\begin{gathered}
\vec{\nabla}\left[\varepsilon_{\mathrm{eff}}(\vec{x}, s) \vec{\nabla}\right] \phi(\vec{x}, s)=-\rho_{\mathrm{ext}}(\vec{x}, s), \\
\vec{E}(\vec{x}, s)=-\vec{\nabla} \phi(\vec{x}, s)
\end{gathered}
$$

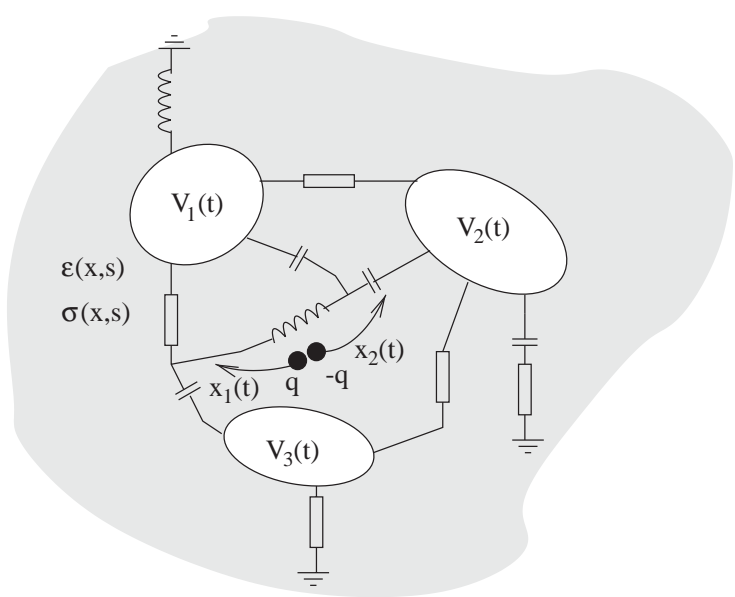

Fig. 1. At $t=0$ a pair of charges is created at a space point from where they move along different trajectories. This movement induces voltages on the electrodes. where $\varepsilon_{\mathrm{eff}}(\vec{x}, s)=\varepsilon(\vec{x}, s)+\sigma(\vec{x}, s) / s$ and $\rho_{\mathrm{ext}}(\vec{x}, s)$ is an 'externally impressed' charge density. This equation is equal to the Poisson equation for electrostatic problems with an effective permittivity, so knowing electrostatic fields for a given permittivity we find the time-depended fields for a scenario with conductive materials by simply replacing $\varepsilon$ by $\varepsilon+\sigma / s$ and performing the inverse Laplace transform. Examples are given in Ref. [3]. For further discussion we can remove the discrete elements since we can consider them to be included in the functions $\varepsilon(\vec{x}, s)$ and $\sigma(\vec{x}, s)$. Before calculating the induced signals we investigate a few properties of the solutions of Eq. (1).

In case we remove the charges, and put the electrodes to potentials $v_{1}(s), v_{2}(s), v_{3}(s)$ we can write the solution as

$\phi_{0}(\vec{x}, s)=\sum_{n=1}^{3} \frac{v_{n}(s)}{V_{0}} \psi_{n}(\vec{x}, s)$

where the $\psi_{n}(\vec{x}, s)$ are the potentials in case electrode $n$ is set to voltage $V_{0}$ and all other electrodes are grounded $\left(\vec{A}_{n}\right.$ indicates the surface of electrode $n$ ):

$$
\begin{array}{r}
\vec{\nabla}\left[\varepsilon_{\mathrm{eff}}(\vec{x}, s) \vec{\nabla}\right] \psi_{n}(\vec{x}, s)=0 \\
\left.\psi_{n}(\vec{x}, s)\right|_{\vec{x}=\vec{A}_{m}}=V_{0} \delta_{n m} .
\end{array}
$$

We call $\psi_{n}(\vec{x}, s)$ the weighting potential of electrode $n$ and $\vec{E}_{n}(\vec{x}, s)=-\vec{\nabla} \psi_{n}(\vec{x}, s)$ the weighting field of electrode $n$ (Fig. 2). The fact that Eq. (2) satisfies the boundary conditions is evident by construction, and since such a solution is unique it is the general solution. The external currents $i_{n}^{\text {ext }}(s)$ that we have to put to the electrodes in order to provide the potentials $v_{n}(s)$ are given by (recalling that differentiation with respect to time corresponds to multiplication with $s$ in the Laplace domain)

$$
\begin{aligned}
i_{n}^{\text {ext }}(s)= & s \int_{V_{n}} \rho_{\text {ext }} \mathrm{d}^{3} x=-s \\
& \times \oint_{\vec{A}_{n}} \varepsilon_{\text {eff }} \vec{\nabla} \phi_{0} \mathrm{~d} \vec{A}=\sum_{m=1}^{3} y_{n m}(s) v_{m}(s)
\end{aligned}
$$




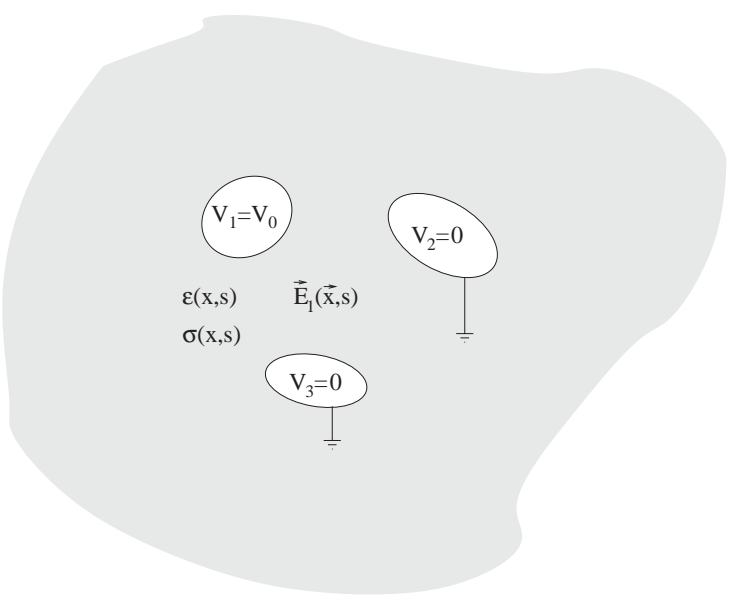

Fig. 2. The weighting potential $\psi_{n}(\vec{x}, s)$ and corresponding weighting field $\vec{E}_{n}(\vec{x}, s)$ are defined by applying a voltage $V_{0}$ (a delta voltage $V_{0} \delta(t)$ in the time domain) to electrode $n$ while grounding the other electrodes.

where we have defined the admittance matrix:

$y_{n m}(s)=\frac{s}{V_{0}} \oint_{\vec{A}_{n}} \varepsilon_{\text {eff }}(\vec{x}, s) \vec{E}_{m}(\vec{x}, s) \mathrm{d} \vec{A}$.

Eq. (4) can be represented by an equivalent circuit diagram with node impedances $Z_{n m}(s)$ (Fig. 3). The current flowing between node $n$ and $m$ is related to the node voltages by $i_{n m}=\left(v_{n}-v_{m}\right) / Z_{n m}$. The sum of all currents at node $n$ is equal to $i_{n}^{\text {ext }}$ i.e. $i_{n}^{\text {ext }}=$ $\sum_{m=1}^{3} i_{n m}$. Comparing these relations to Eq. (4) defines the relation between $Z_{n m}$ and $y_{n m}$ as

$$
\begin{aligned}
& Z_{n m}(s)=-\frac{1}{y_{n m}(s)} \quad n \neq m, \\
& Z_{n n}(s)=\frac{1}{\sum_{m=1}^{3} y_{n m}(s)} .
\end{aligned}
$$

Finally we want to define the potentials $\chi_{n}(\vec{x}, s)$ and related fields $\vec{K}_{n}(\vec{x}, s)=-\vec{\nabla} \chi_{n}(\vec{x}, s)$ by putting a delta current $i_{n}^{\operatorname{ext}}(t)=I_{0} \delta(t)$ to electrode $n$ and zero current on the other electrodes. This corresponds to putting a charge of $q_{n}^{\text {ext }}(t)=I_{0}$ to the electrode, which in the Laplace domain reads as $q_{n}^{\mathrm{ext}}(s)=I_{0} / s$ :

$$
\begin{aligned}
& \vec{\nabla}\left[\varepsilon_{e f f}(\vec{x}, s) \vec{\nabla}\right] \chi_{n}(\vec{x}, s)=0, \\
& \quad-\oint_{A_{n}} \varepsilon_{\mathrm{eff}}(\vec{x}, s) \vec{\nabla} \chi_{m}(\vec{x}, s) \mathrm{d} \vec{A}=\frac{I_{0}}{s} \delta_{n m} .
\end{aligned}
$$

The current will cause voltages $v_{m}(s)=y_{n m}^{-1}(s) I_{0}$ on the electrodes, for which we know the general solution from Eq. (2), so we have the relation

$\chi_{n}(\vec{x}, s)=\frac{I_{0}}{V_{0}} \sum_{m=1}^{3} y_{n m}^{-1}(s) \psi_{m}(\vec{x}, s)$.

We will use all these relations in the next section.

\section{Induced signals}

The voltages $V_{n}(t)$ induced by the movement of the charges (Fig. 1) are formally given by the relations ( $L$ denotes the Laplace transform):

$$
\begin{gathered}
\rho_{q}(\vec{x}, s)=L\left[q \delta^{3}\left(\vec{x}-\vec{x}_{1}(t)\right)\right. \\
\left.-q \delta^{3}\left(\vec{x}-\vec{x}_{2}(t)\right)\right] \\
\vec{\nabla}\left[\varepsilon_{\mathrm{eff}}(\vec{x}, s) \vec{\nabla}\right] \phi(\vec{x}, s)=-\rho_{q}(\vec{x}, s), \\
V_{n}(s)=\left.\phi(\vec{x}, s)\right|_{\vec{x}=\vec{A}_{n}} .
\end{gathered}
$$

We can now simplify these relations by using an extended version of Green's second theorem:

$$
\begin{aligned}
\oint_{\vec{A}} & {[\psi(\vec{x}) f(\vec{x}) \vec{\nabla} \phi(\vec{x})-\phi(\vec{x}) f(\vec{x}) \vec{\nabla} \psi(\vec{x})] \mathrm{d} \vec{A} } \\
= & \int_{V}[\psi(\vec{x}) \vec{\nabla}[f(\vec{x}) \vec{\nabla}] \phi(\vec{x})-\phi(\vec{x}) \vec{\nabla} \\
& \times[f(\vec{x}) \vec{\nabla}] \psi(\vec{x})] \mathrm{d}^{3} x
\end{aligned}
$$

where the surface $\vec{A}$ encloses the volume $V$. This relation holds for arbitrary functions $\phi(\vec{x}), \psi(\vec{x})$ and $f(\vec{x})$. We insert $\phi(\vec{x}, s)$ from Eq. (10), replace $\psi(\vec{x}, s)$ by $\chi_{n}(\vec{x}, s)$ from Eq. (7) and $f(\vec{x})=\varepsilon_{\text {eff }}(\vec{x}, s)$. We assume $V$ to be the entire volume between the electrodes and $\vec{A}$ to be the enveloping surface, i.e., the surfaces of the electrodes and a 'grounded' surface at infinity, which yields:

$$
V_{n}(s)=\frac{s}{I_{0}} \int_{V} \chi_{n}(\vec{x}, s) \rho_{q}(\vec{x}, s) \mathrm{d}^{3} x .
$$

Inserting the charge density (Eq. (9)), and returning to the time domain gives

$$
\begin{aligned}
V_{n}(t)= & -\frac{q}{I_{0}} \int_{0}^{t} \vec{K}_{n}\left[\vec{x}_{1}\left(t^{\prime}\right), t-t^{\prime}\right] \overrightarrow{\dot{x}}_{1}\left(t^{\prime}\right) \mathrm{d} t^{\prime} \\
& +\frac{q}{I_{0}} \int_{0}^{t} \vec{K}_{n}\left[\vec{x}_{2}\left(t^{\prime}\right), t-t^{\prime}\right] \overrightarrow{\dot{x}}_{2}\left(t^{\prime}\right) \mathrm{d} t^{\prime}
\end{aligned}
$$


where $\overrightarrow{\dot{x}}(t)=\mathrm{d} / \mathrm{d} t \vec{x}(t)$ is the velocity vector of the charge. The voltages $V_{n}(t)$ can therefore be calculated by the time-dependent fields $\vec{K}_{n}(\vec{x}, t)$. There is however a more practical way to proceed: if we imagine the electrodes to be grounded (Fig. 4), the voltages $V_{n}(t)$ are always zero and the movement of the charges induces currents that flow between the electrodes and ground. Formally, we can find this current by solving the equations:

$$
\begin{gathered}
\vec{\nabla}\left[\varepsilon_{\mathrm{eff}}(\vec{x}, s) \vec{\nabla}\right] \phi(\vec{x}, s)=-\rho_{q}(\vec{x}, s), \\
\left.\phi(\vec{x}, s)\right|_{\vec{x}=\vec{A}_{n}}=0
\end{gathered}
$$

and calculating

$$
\begin{aligned}
I_{n}(s)= & -s Q_{n}(s)=s \\
& \times \int_{\vec{A}_{n}} \varepsilon_{\text {eff }}(\vec{x}, s) \vec{\nabla} \phi(\vec{x}, s) \mathrm{d} \vec{A} .
\end{aligned}
$$

Using again Green's theorem and inserting Eqs. (15) and (16) and $\psi_{n}$ from Eq. (3) we find:

$I_{n}(s)=\frac{s}{V_{0}} \int_{V} \psi_{n}(\vec{x}, s) \rho_{q}(\vec{x}, s) \mathrm{d}^{3} x$

which in the time domain reads as

$$
\begin{aligned}
I_{n}(t)= & -\frac{q}{V_{0}} \int_{0}^{t} \vec{E}_{n}\left[\vec{x}_{1}\left(t^{\prime}\right), t-t^{\prime}\right] \overrightarrow{\dot{x}}_{1}\left(t^{\prime}\right) \mathrm{d} t^{\prime} \\
& +\frac{q}{V_{0}} \int_{0}^{t} \vec{E}_{n}\left[\vec{x}_{2}\left(t^{\prime}\right), t-t^{\prime}\right] \overrightarrow{\dot{x}}_{2}\left(t^{\prime}\right) \mathrm{d} t^{\prime} .
\end{aligned}
$$

We can now rewrite Eq. (13) by inserting Eqs. (8) and (17), which yields

$$
\begin{aligned}
V_{n}(s)= & \sum_{m=1}^{3} y_{n m}^{-1}(s) \frac{1}{V_{0}} \\
& \times \int_{V} \psi_{m}(\vec{x}, s) s \rho_{q}(\vec{x}, s) \mathrm{d}^{3} x \\
= & \sum_{m=1}^{3} y_{n m}^{-1}(s) I_{m}(s) .
\end{aligned}
$$

The currents induced on the grounded electrodes are related to the induced voltages by the admittance matrix and we can therefore make use of the equivalent circuit diagram shown before.

\section{Extended theorems}

We want to summarize the relations found in the last section:

The voltage $V_{n}(t)$ induced on electrode $n$, as shown in Fig. 1, can be calculated by removing the charges, putting a delta current $I_{0} \delta(t)$ to the electrode in question (leaving the discrete elements in place), which defines a time-dependent field $\vec{K}_{n}(\vec{x}, t)$ in the space between the electrodes, from which the induced voltage can be calculated with Eq. (14).

A more practical way to calculate the signals is given by proceeding in four steps:

(i) One removes the charges and discrete elements, and calculates the weighting fields $\vec{E}_{n}(\vec{x}, t)$, which are defined by applying the voltage $V_{0} \delta(t)$ to an electrode while grounding all others (Fig. 2 and Eq. (3)).

(ii) One finds the equivalent circuit diagram of the electrode system by calculating the admittance matrix and the related impedance elements (Fig. 3, Eqs. (5) and (6)).

(iii) Next, one calculates the currents $I_{n}(t)$ induced on the electrodes in case they are grounded (Fig. 4 and Eq. (18)).

(iv) Adding this equivalent circuit to the discrete elements and putting the calculated currents $I_{n}(t)$ as ideal current sources to the electrode nodes (Fig. 5) finally gives the induced voltages $V_{n}(t)$.

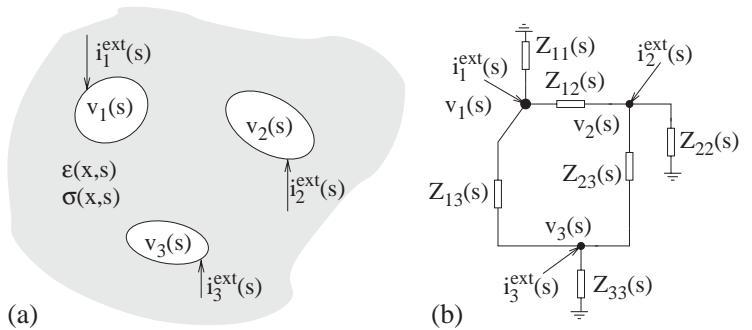

Fig. 3. The influence of the medium can be represented by an equivalent circuit diagram. In case the medium is insulating the impedance elements are the mutual capacitances of the electrodes. 


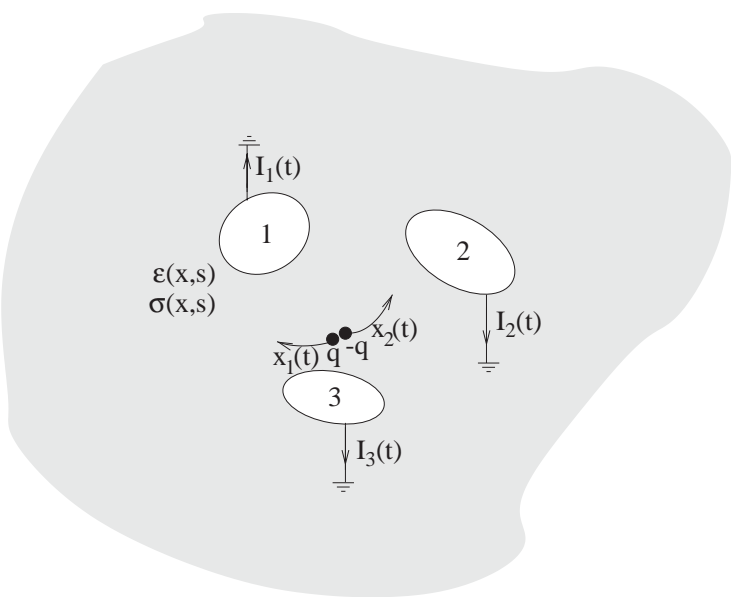

Fig. 4. In case the electrodes are grounded the movement of the charges induces currents that flow between the electrodes and ground.

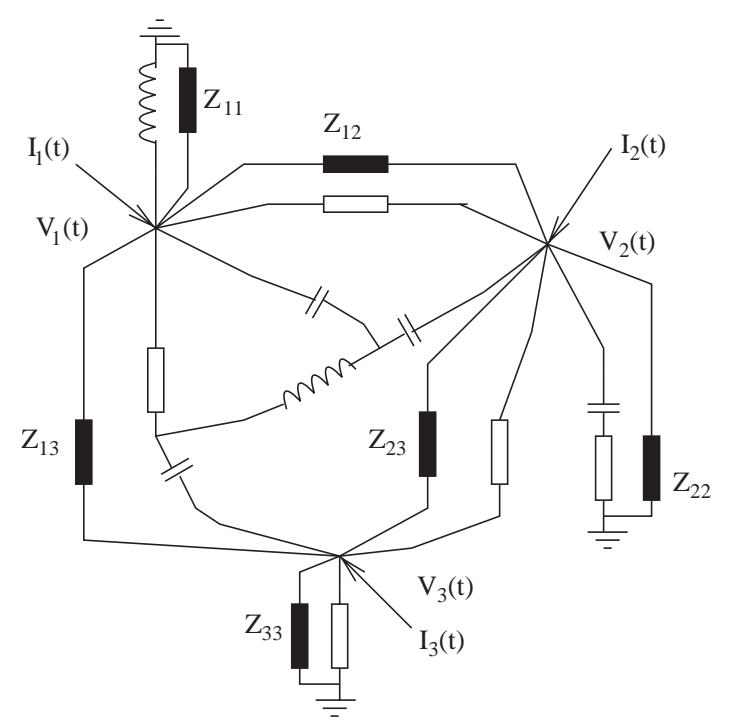

Fig. 5. Circuit diagram defining the voltages $V_{n}(t)$.

\section{Example}

As an example we investigate a structure shown in Fig. 6 showing a parallel plate detector containing a conductive layer.

As a first step we calculate the weighting fields. Setting electrode 2 to voltage $V_{0}$ and grounding

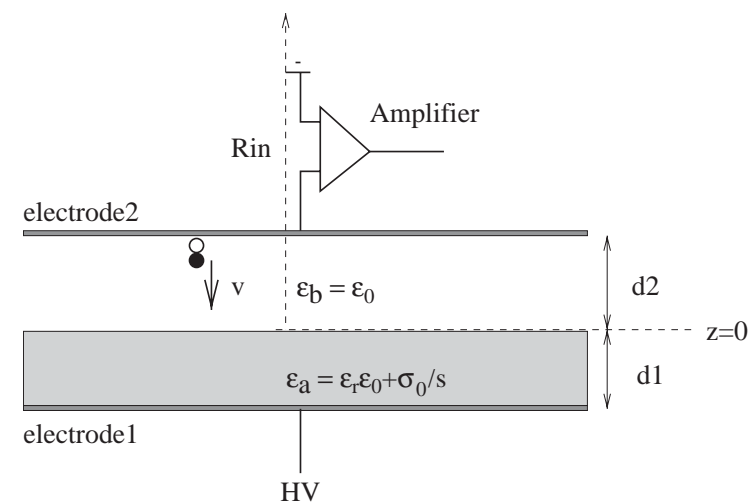

Fig. 6. A geometry representing a resistive plate chamber or underdepleted silicon detector.

electrode 1 we find (in the Laplace domain):

$$
\begin{aligned}
E_{2 z}(s)= & -\frac{\varepsilon_{a} V_{0}}{\varepsilon_{a} d_{2}+\varepsilon_{b} d_{1}}=-\frac{V_{0} \varepsilon_{r}}{\left(d_{1}+d_{2} \varepsilon_{r}\right)} \\
& \times \frac{s+1 / \tau_{1}}{s+1 / \tau_{2}}, \quad z>0 \\
= & -\frac{\varepsilon_{b} V_{0}}{\varepsilon_{a} d_{2}+\varepsilon_{b} d_{1}}=-\frac{V_{0}}{\left(d_{1}+d_{2} \varepsilon_{r}\right)} \\
& \times \frac{s}{s+1 / \tau_{2}}, \quad z<0 \\
& \tau_{1}=\frac{\varepsilon_{r} \varepsilon_{0}}{\sigma}, \quad \tau_{2}=\frac{\varepsilon_{0}}{\sigma}\left(\frac{d_{1}+d_{2} \varepsilon_{r}}{d_{2}}\right) .
\end{aligned}
$$

The weighting field of the other electrode is given by $E_{1}(s)=-E_{2}(s)$, so we have $I_{1}(t)=-I_{2}(t)$. Using the particle trajectory $z(t)=d_{2}-v t, t<T=$ $d_{2} / v$ and evaluating Eq. (18) we find the induced current of

$$
\begin{aligned}
I_{2}(t) & =-q v \frac{\varepsilon_{r}}{d_{1}+\varepsilon_{r} d_{2}}\left[1+\frac{d_{1}}{d_{2} \varepsilon_{r}}\left(1-\mathrm{e}^{-t / \tau_{2}}\right)\right], t<T \\
& =-q v \frac{1}{d_{1}+\varepsilon_{r} d_{2}} \frac{d_{1}}{d_{2}}\left(\mathrm{e}^{T / \tau_{2}}-1\right) \mathrm{e}^{-t / \tau_{2}}, t>T .
\end{aligned}
$$

The current is shown in Fig. 7. For this example we assumed $d_{1}=3 \mathrm{~mm}, d_{2}=0.3 \mathrm{~mm}, \varepsilon_{r}=6$. We also define $\tau=\varepsilon_{0} / \sigma$. If the resistivity of the layer is very high $(\tau \gg T)$, a constant current is induced while the charge is moving; once the charge hits 


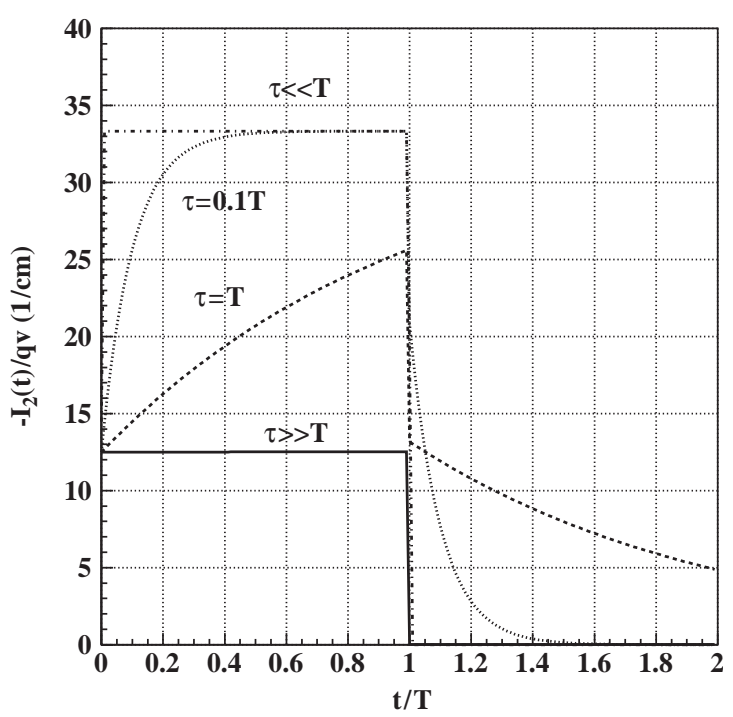

Fig. 7. Induced currents for different values of $\sigma$.

the surface the current is zero. In case $(\tau=T)$ the induced current is not only caused by the movement of the charge but in addition by the flow of charges in the conductive layer. Once the charge hits the surface it is 'compensated' with a time constant $\tau$ by charges flowing in the layer. In case the layer is very conductive $\tau \ll T$ it acts like a metal plate and the induced current is equal to the one in a parallel plate geometry of plate separation $d_{2}$. In common RPCs made of glass or Bakelite, the typical resistivites $\rho=1 / \sigma$ are $10^{10}-10^{12} \Omega \mathrm{cm}$ giving time constants $\tau \approx 1-100 \mathrm{~ms}$. Since the electron movement time is of the order $T \approx$ few nanoseconds, we always have the condition $\tau \gg T$ in this detectors. Undepleted silicon with doping concentration for e.g. $N_{\mathrm{A}}=10^{12} / \mathrm{cm}^{3}$ has a volume resistivity of $1 / \sigma \approx 5 \mathrm{k} \Omega \mathrm{cm}$ which gives a times constant of about $0.5 \mathrm{~ns}$. Therefore, we usually have the case $\tau \ll T$ in these conditions. Evaluating Eqs. (5) and (6) and assuming a detector area $A$ we find the impedance elements $Z_{11}=Z_{22}=\infty$ and

$Z_{12}(s)=\frac{1}{s C_{1}}+\frac{R / s C_{2}}{R+1 / s C_{2}}$

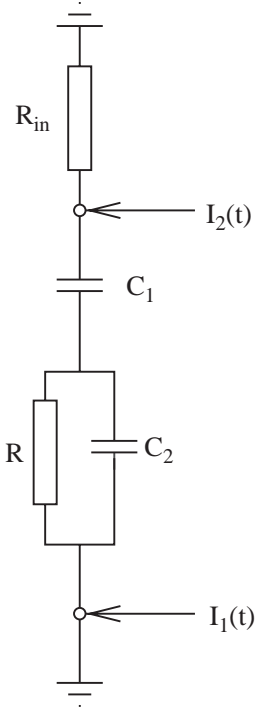

Fig. 8. Equivalent circuit for the geometry in Fig. 6.

with

$C_{1}=\varepsilon_{0} \frac{A}{d_{2}}, \quad C_{2}=\varepsilon_{r} \varepsilon_{0} \frac{A}{d_{1}}, \quad R=\frac{1}{\sigma} \frac{d_{1}}{A}$.

This is a very intuitive result: the geometry in Fig. 6 is equal to a capacitor in series with the parallel circuit of a capacitor and a resistor. In order to arrive at the induced voltages, we finally connect the equivalent circuit to the discrete elements and add the signals as ideal current sources (Fig. 8). This step is usually performed by an analog circuit simulator program.

\section{Summary}

We presented theorems that allow the calculation of induced signals in particle detectors containing materials with finite conductivity. The time dependent weighting fields of the electrodes can be obtained from electrostatic solutions (in the Laplace domain) by simply replacing $\varepsilon$ with $\varepsilon+$ $\sigma / s$. From these weighting fields one obtains the currents induced on grounded electrodes, the equivalent circuit diagram of the detector and finally the induced voltages. An application of the theorems to resistive plate chambers and silicon detectors was presented. 


\section{Erratum}

In Ref. [3] there are sign errors in the final formulas. Eq. (41) of Ref. [3] should read like: $\left.\quad \mathrm{V}_{1}(\mathrm{t})=\frac{Q}{q_{0}} \psi_{\mathrm{V}}\left(\vec{x}_{0}(t), t\right)-\frac{Q}{q_{0}} \int_{0}^{t} \vec{E}_{V} \overrightarrow{(}_{x_{0}}\left(t^{\prime}\right), t-t^{\prime}\right)$ $\vec{x}_{0}\left(t^{\prime}\right) d t^{\prime}$ Eqs. (43) and (44) of Ref. [3] should be replaced by Eqs. (14) and (18) from this publication.

\section{References}

[1] S. Ramo, Proc. IRE 27 (1939) 584.

[2] E. Gatti, G. Padovini, V. Radeka, Nucl. Instr. and Meth. 193 (1982) 651.

[3] W. Riegler, Nucl. Instr. and Meth. A 491 (2002) 258.

[4] H.A. Haus, J.R. Melcher, Electromagentic Fields and Energy, Prentice-Hall, Englewood Cliffs, NJ, 1989.

[5] T. Heubrandtner, B. Schnizer, Nucl. Instr. and Meth. A 478 (2002) 444. 